\title{
LETTERS
}

\section{Fully reversible pulmonary arterial hypertension associated with dasatinib treatment for chronic myeloid leukaemia}

\section{To the Editors:}

Pulmonary arterial hypertension (PAH) is a devastating disease that is characterised by a progressive increase in pulmonary vascular resistance (PVR), chronic right heart failure and premature death. Pulmonary hypertension $(\mathrm{PH})$ is defined by a mean pulmonary artery pressure $\left(\bar{P}_{\mathrm{pa}}\right)$ $\geqslant 25 \mathrm{mmHg}$ at rest, and the haemodynamic definition discerns pre- and post-capillary PH [1]. Furthermore, PAH is distinguished from other forms of $\mathrm{PH}$ that may occur secondary to various underlying causes, including left heart disease, chronic lung disorders and thromboembolic disease [1]. Although the development of targeted PAH therapies, such as endothelin receptor antagonists, phosphodiesterase type- 5 inhibitors (PDE5i) and prostanoids, has markedly improved the clinical situation and outcome of affected patients, the current medical treatment of PAH is not satisfactory.

Recently, tyrosine kinase inhibitors (TKIs), particularly imatinib, have been proposed as a potential novel treatment option in PAH [2-7]. Imatinib was the first TKI that achieved regulatory approval for the treatment of chronic myeloid leukaemia (CML) and has become the standard of care for patients with this disease, as it inhibits the hybrid $B C R-A B L$ oncogene that is pathogenic in the development of CML. Imatinib inhibits several tyrosine kinases, including BCR-ABL, the platelet-derived growth factor receptor (PDGFR) and c-Kit. More recently, additional TKIs, including dasatinib and nilotinib, have been introduced for the treatment of CML in patients who are intolerant or unresponsive to imatinib.

The potential efficacy of imatinib in PAH is attributable to its inhibitory effect on the PDGFR. Platelet-derived growth factor (PDGF) is thought to play a pivotal role in the pathobiology of $\mathrm{PAH}$ by initiating and maintaining the underlying pulmonary vascular remodelling [7, 8]. Consistent with this, inhibition of PDGFR signalling by imatinib was recently shown to reverse $\mathrm{PAH}$ in animal models, and to improve the clinical situation in patients with severe $\mathrm{PAH}$ and inadequate response to established therapy [2-7]. Herein, we report the development of severe $\mathrm{PAH}$ associated with dasatinib treatment for CML, which completely resolved after termination of this TKI.

A 47-yr-old male presented with a 3-month history of progressive exertional dyspnoea (World Health Organization (WHO) functional class III), fatigue and dizziness. He was known to suffer from a Philadelphia chromosome-positive $\mathrm{CML}$ with b3a2 and b2a2 BCR-ABL transcripts. The patient did not have any other significant diseases. In particular, there were no known cardiac or respiratory conditions. At the time of presentation, CML had been medically treated for 7 yrs by the use of various compounds. The initial treatment consisted of a combination of PEGylated interferon- $\alpha$ and imatinib. This regimen had to be discontinued after $1 \mathrm{yr}$ because of a significant liver enzyme elevation and recurrent exanthema. A rechallenge had produced the same side-effects. At that point, CML treatment was switched to dasatinib $\left(70 \mathrm{mg} \cdot \mathrm{day}^{-1}\right.$ b.i.d.), with several dose adjustments over time because of recurrent pleural effusions that were first documented 13 months after initiation of dasatinib. At the time of presentation, the patient had been on a stable dasatinib dose of $100 \mathrm{mg} \cdot \mathrm{day}^{-1}$ for 39 months.

On admission, physical examination revealed a systolic heart murmur, a resting tachycardia (heart rate 100 beats $\cdot \mathrm{min}^{-1}$ ) with normal blood pressure (120/85 $\mathrm{mmHg})$, jugular venous distention, hepatomegaly and peripheral oedema. Electrocardiography showed right axis deviation, a right bundle branch block and T-wave inversions in leads V1-4. The patient had grade 3 bilateral pleural effusions requiring thoracentesis and pleural fluid analysis was consistent with an exudate. Transthoracic echocardiography revealed regularly sized left heart chambers and left ventricular ejection fraction (LVEF) was normal. However, right heart cavities were enlarged (right atrial area $22 \mathrm{~cm}^{2}$; basal right ventricular end-diastolic diameter $51 \mathrm{~mm}$ ) and a mild pericardial effusion was detected. The systolic pulmonary artery pressure $(P$ pa $)$ was estimated at $61 \mathrm{mmHg}$ and right ventricular function was impaired, as assessed by a reduced tricuspid annular plane systolic excursion of $16 \mathrm{~mm}$. The patient was subjected to right heart catheterisation, which demonstrated severe pre-capillary $\mathrm{PH}$. There was a marked elevation of the $\bar{P}_{\text {pa }}$ to $60 \mathrm{mmHg}$, pulmonary capillary wedge pressure was normal $(11 \mathrm{mmHg})$, and cardiac output $(\mathrm{CO})$ was reduced to $3.6 \mathrm{~L} \cdot \mathrm{min}^{-1}$. Consequently, the calculated PVR was elevated at 13.6 Wood units. The patient was unresponsive to vasoreactivity testing with inhaled iloprost.

Potential other causes of $\mathrm{PH}$, such as pulmonary embolism or parenchymal lung disease, were ruled out by contrastenhanced spiral computed tomography and a ventilation/ perfusion scan. Consistent with signs of right ventricular strain, laboratory testing showed significantly elevated $\mathrm{N}$ terminal pro-brain natriuretic peptide serum levels $(2,888$ $\mathrm{ng} \cdot \mathrm{L}^{-1}$ ) and the 6-min walk distance was limited to $435 \mathrm{~m}$. Furthermore, laboratory screening tests for HIV and for systemic rheumatic diseases, including antinuclear antibodies, 
antibodies to double-stranded DNA and anti-Scl-70 antibodies were negative. Concurrent administration of drugs or toxins suspected to play a predisposing or facilitating role in the development of PAH was ruled out. Due to previously reported clues for dasatinib-related development of PAH [9-11], CML treatment with dasatinib was permanently discontinued and a targeted PAH therapy with the PDE5i sildenafil was initiated. Subsequently, the patient underwent close follow-up investigations.

Re-evaluation after 2 months revealed a significant improvement of the clinical condition to WHO functional class II and right heart catheterisation evidenced a marked decrease in $P$ pa, accompanied by a substantial increase in $\mathrm{CO}$ and a related decrease in PVR. Since the patient's leukaemia required further treatment, the alternate TKI nilotinib was started at a dose of $400 \mathrm{mg}$ b.i.d. Subsequently, repeat noninvasive evaluations showed normalisation of all measured parameters, such that targeted PAH therapy with sildenafil was stopped after 6 months. A third right-heart catheterisation was performed

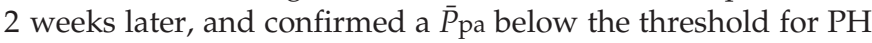
and even for borderline $\mathrm{PH}[1,12]$. The other haemodynamic parameters had improved accordingly. This coincided with complete resolution of breathlessness. To date, 3 months after discontinuation of specific PAH therapy, the patient has remained asymptomatic with no evidence of recurrent $\mathrm{PAH}$ or pleural effusions and was continued on nilotinib with close monitoring. The details of the invasive and noninvasive diagnostic work-up for this patient are shown in table 1 .

The case we present here is consistent with recent reports on transient PAH in conjunction with pleural effusion secondary to

\begin{tabular}{|c|c|c|c|c|c|c|}
\hline \multirow[t]{3}{*}{ TABLE 1} & \multicolumn{6}{|c|}{$\begin{array}{l}\text { Invasive and noninvasive evaluation during the } \\
\text { course of the disease: display of key parameters }\end{array}$} \\
\hline & \multirow[t]{2}{*}{ Baseline } & \multicolumn{5}{|c|}{ Time months } \\
\hline & & 1 & 2 & 4 & 6 & 9 \\
\hline $\begin{array}{l}\text { Systolic Ppa } \\
\mathrm{mmHg}\end{array}$ & 82 & & 45 & & 39 & \\
\hline $\begin{array}{l}\text { Diastolic Ppa } \\
\mathrm{mmHg}\end{array}$ & 44 & & 17 & & 11 & \\
\hline $\bar{P}$ pa $\mathrm{mmHg}$ & 60 & & 28 & & 20 & \\
\hline Ppcw mmHg & 11 & & 7 & & 7 & \\
\hline $\bar{P}_{\text {ra }} \mathrm{mmHg}$ & 20 & & 4 & & 3 & \\
\hline $\mathrm{CO} L \cdot \mathrm{min}^{-1}$ & 3.6 & & 4.8 & & 6.3 & \\
\hline PVR Wood units & 13.6 & & 4.4 & & 2.0 & \\
\hline $\begin{array}{l}\text { NT-proBNP } \\
\text { ng } \cdot L^{-1}\end{array}$ & 2888 & 1.037 & 282 & 72 & 50 & 25 \\
\hline 6MWD m & 435 & 550 & 600 & 600 & 600 & 600 \\
\hline RVEDD mm & 51 & 44 & 47 & 38 & 35 & 35 \\
\hline TAPSE mm & 16 & 17 & 18 & 22 & 21 & 25 \\
\hline
\end{tabular}

Ppa: pulmonary artery pressure; $\bar{P}$ pa: mean $P$ pa; $P$ pcw: pulmonary capillary wedge pressure; $\bar{P}_{\text {ra: }}$ mean right atrial pressure; CO: cardiac output; PVR: pulmonary vascular resistance; NT-proBNP: N-terminal pro-brain natriuretic peptide; 6MWD: 6-min walk distance; RVEDD: right ventricle end-diastolic diameter; TAPSE: tricuspid annular plane systolic excursion. the TKI dasatinib that have been described in a few patients [9-11]. In all cases, dasatinib had been given for a rather long period of time (18-60 months) before PAH developed and all patients presented with pleural effusions. Notably, these patients also demonstrated a rapid onset of symptoms and reversal of pulmonary vasculopathy after termination of dasatinib, leading to haemodynamic normalisation (table 2). This is remarkable because complete remission and normalisation of pulmonary hemodynamics is usually not achieved in PAH by medical treatment. In the present case, pulmonary haemodynamics had normalised 6 months after the onset of $\mathrm{PAH}$, even after termination of PAH therapy. Therefore, it is assumed that the pathophysiological mechanism leading to increased PVR and PAH differed from the vascular alterations typically seen in $\mathrm{PAH}$.

The ability to inhibit PDGFR signalling suggests similar effects of imatinib and dasatinib on pulmonary vascular remodelling. However, while imatinib was effective for the treatment of $\mathrm{PAH}$ in several cases and a recent phase II study [3-7], it appears that dasatinib is surprisingly associated with the

\section{TABLE 2 Comparison of haemodynamic evolution in the case reports previously described in the literature and the presented case}

Baseline

Time months

3

6

\begin{tabular}{|c|c|c|c|c|c|}
\hline \multicolumn{6}{|c|}{ Systolic Ppa mmHg } \\
\hline RASHEED [10] & $70+$ Pra $^{\#}$ & $31+$ Pra $^{\#}$ & & & \\
\hline MATTEI [11] & 66 & & 48 & & 40 \\
\hline Present case & 82 & 45 & & 39 & \\
\hline \multicolumn{6}{|l|}{ Diastolic Ppa } \\
\hline \multicolumn{6}{|l|}{$\mathrm{mmHg}$} \\
\hline \multicolumn{6}{|l|}{ RASHEED [10] } \\
\hline MATtEI [11] & 28 & & 19 & & 17 \\
\hline Present case & 44 & 17 & & 11 & \\
\hline \multicolumn{6}{|l|}{$\bar{P}_{\text {pa }} \mathrm{mmHg}$} \\
\hline \multicolumn{6}{|l|}{ RASHEED [10] } \\
\hline MATTEI [11] & 40 & & 29 & & 25 \\
\hline Present case & 60 & 28 & & 20 & \\
\hline \multicolumn{6}{|l|}{ Ppcw mmHg } \\
\hline \multicolumn{6}{|l|}{ RASHEED [10] } \\
\hline \multicolumn{6}{|l|}{ MATTEI [11] } \\
\hline Present case & 11 & 7 & & 7 & \\
\hline \multicolumn{6}{|l|}{$\mathrm{CO} L \cdot \mathrm{min}^{-1}$} \\
\hline \multicolumn{6}{|l|}{ RASHEED [10] } \\
\hline MATTEI [11] & 3.9 & & 6.47 & & 7.23 \\
\hline Present case & 3.6 & 4.8 & & 6.3 & \\
\hline \multicolumn{6}{|c|}{ PVR Wood units } \\
\hline \multicolumn{6}{|l|}{ RASHEED [10] } \\
\hline MATTEI [11] & 8.2 & & 7.23 & & 2.3 \\
\hline Present case & 13.6 & 4.4 & & 2.0 & \\
\hline
\end{tabular}

Ppa: pulmonary artery pressure; $\bar{P}$ pa: mean $P$ pa; Ppcw: pulmonary capillary wedge pressure; CO: cardiac output; PVR: pulmonary vascular resistance; Pra: right atrial pressure. ${ }^{\#}$ : systolic Ppa estimated by echocardiography. 
onset of PAH, at least in some patients. This raises questions about the underlying mechanism and the differences between dasatinib and other TKIs. Dasatinib is a multitarget kinase inhibitor of BCR-ABL, the PDGFR, Src, and c-Kit, which is structurally distinct from imatinib, has a broader spectrum of activity and $\sim 300$-fold greater potency than imatinib in in vitro studies. Global chemical proteomic profiling of imatinib, dasatinib and nilotinib identified novel kinase and nonkinase targets for the three TKIs with only a small overlap covering the ABL kinases [13]. Imatinib and nilotinib displayed much more specific target profiles compared with dasatinib, suggesting that dasatinib has a broad range of activity in multiple biological processes. The main known difference in the kinase profile between dasatinib and other TKIs, such as imatinib or nilotinib, is the additional inhibition of Src family kinases. It may therefore be speculated that Src inhibition may play a role in the development of dasatinib-associated PAH. It has recently been reported that dasatinib inhibits PDGF-induced proliferation and migration of vascular smooth muscle cells via inhibition of both PDGFR activity and PDGF-dependent Src activation downstream of the PDGFR [14], which may be beneficial in PAH. However, Src family kinases are required for degradation of activated PDGFRs [15]. Hence, inhibition of Src may result in increased signalling by PDGF and possibly other growth factors. An additional explanation may be related to off-target kinase inhibition. These mechanisms may affect pulmonary vascular wall integrity, possibly leading to (peri)vascular oedema and subsequent PVR increase.

The clear association of pleural effusions and PAH with dasatinib intake and their resolution upon discontinuation of dasatinib suggest a causal role of the drug in disease development. Pleural effusions occur in 17-35\% (grades 3-4 in 4\%) of dasatinib-treated patients [9], but were not found in individuals treated with imatinib or nilotinib. An increase in Ppa was detected at the onset of pleural effusions in a subset of CML patients treated with dasatinib, suggesting the possibility of drug-related $\mathrm{PAH}$ [9-11]. A potential concern with TKIs, particularly imatinib, is cardiotoxicity, which is attributable to their inhibitory effect on c-Abl [16]. However, LVEF was normal in all reported cases of dasatinib-associated $\mathrm{PAH}$, and the fact that most pleural effusions were exudates also argues against primary cardiac impairment but indicates an alternative pathological mechanism. The occurrence of PAH with pleural effusions at a late onset (26 months in our patient) suggests a chronic pathological mechanism with an insidious onset rather than an acute inflammatory or cardiac aetiology. Transient parenchymal changes in the lung have been described in patients on dasatinib therapy, all of which resolved after drug withdrawal [17].

In conclusion, chronic treatment with dasatinib may be complicated by severe, albeit reversible $\mathrm{PAH}$ and right ventricular failure that occur concurrent with pleural effusions. The incidence of dasatinib-associated $\mathrm{PAH}$ and the underlying molecular mechanisms should be elucidated in future studies.

\section{Dumitrescu, C. Seck, H. ten Freyhaus, F. Gerhardt, E. Erdmann and S. Rosenkranz}

Klinik III für Innere Medizin, Herzzentrum der Universität zu Köln, Köln, Germany.
Correspondence: S. Rosenkranz, Klinik III für Innere Medizin, Herzzentrum der Universität zu Köln, Kerpener Str. 62, 50937 Köln, Germany. E-mail: stephan.rosenkranz@uk-koeln.de

Statement of Interest: None declared.

\section{REFERENCES}

1 Galiè N, Hoeper MM, Humbert M, et al. Guidelines for the diagnosis and treatment of pulmonary hypertension: the Task Force for the Diagnosis and Treatment of Pulmonary Hypertension of the European Society of Cardiology (ESC) and the European Respiratory Society (ERS), endorsed by the International Society of Heart and Lung Transplantation (ISHLT). Eur Respir J 2009; 34: 1219-1263.

2 Schermuly RT, Dony E, Ghofrani HA, et al. Reversal of experimental pulmonary hypertension by PDGF inhibition. J Clin Invest 2005; 115: 2811-2821.

3 Ghofrani HA, Seeger W, Grimminger F. Imatinib for the treatment of pulmonary arterial hypertension. N Engl J Med 2005; 353: 1412-1413.

4 Patterson KC, Weissmann A, Ahmadi T, et al. Imatinib mesylate in the treatment of refractory idiopathic pulmonary arterial hypertension. Ann Intern Med 2006; 145: 152-153.

5 Souza R, Sitbon O, Parent G, et al. Long term imatinib treatment in pulmonary arterial hypertension. Chest 2006; 61: 736.

6 ten Freyhaus H, Dumitrescu D, Bovenschulte H, et al. Significant improvement of right ventricular function by imatinib mesylate in scleroderma-associated pulmonary arterial hypertension. Clin Res Cardiol 2009; 98: 265-267.

7 Ghofrani HA, Morrell NW, Hoeper MM, et al. Imatinib in pulmonary arterial hypertension patients with inadequate response to established therapy. Am J Respir Crit Care Med 2010; 182: 1171-1177.

8 Perros F, Montani D, Dorfmüller P, et al. Platelet-derived growth factor expression and function in idiopathic pulmonary arterial hypertension. Am J Respir Crit Care Med 2008; 178: 81-88.

9 Quinta's-Cardama A, Kantarjian H, O'Brien S, et al. Pleural effusion in patients with chronic myelogenous leukemia treated with dasatinib after imatinib failure. J Clin Oncol 2007; 25: 3908-3914.

10 Rasheed W, Flaim B, Seymour JF. Reversible severe pulmonary hypertension secondary to dasatinib in a patient with chronic myeloid leukemia. Leuk Res 2009; 33: 861-864.

11 Mattei D, Feola M, Orzan F, et al. Reversible dasatinib-induced pulmonary arterial hypertension and right ventricle failure in a previously allografted CML patient. Bone Marrow Transplant 2009; 43: 967-968.

12 Kovacs G, Berghold A, Scheidl S, et al. Pulmonary arterial pressure during rest and exercise in healthy subjects: a systematic review. Eur Respir J 2009; 34: 888-894.

13 Rix U, Hantschel O, Durnberger G, et al. Chemical proteomic profiles of the BCR-ABL inhibitors imatinib, nilotinib, and dasatinib reveal novel kinase and nonkinase targets. Blood 2007; 110: 4055-4063.

14 Chen Z, Lee F, Bhalla K, et al. Potent inhibition of platelet-derived growth factor-induced responses in vascular smooth muscle cells by bms-354825 (dasatinib). Mol Pharmacol 2006; 69: 1527-1533.

15 Rosenkranz S, Ikuno Y, Leong FL, et al. Src family kinases negatively regulate platelet-derived growth factor $\alpha$ receptor-dependent signaling and disease progression. J Biol Chem 2000; 275: 9620-9627.

16 Kerkelä R, Grazette L, Yacobi R, et al. Cardiotoxicity of the cancer therapeutic agent imatinib mesylate. Nat Med 2006; 12: 908-916.

17 Bergeron A, Rea D, Levy V, et al. Lung abnormalities after dasatinib treatment for chronic myeloid leukemia: a case series. Am J Respir Crit Care Med 2007; 176: 814-818.

DOI: $10.1183 / 09031936.00154210$ 